RESEARCH REPORT

\title{
EFFECTIVENESS OF LUMBAR STABILIZATION EXERCISES IN NON-SPECIFIC LOW BACK PAIN AMONG OCCUPATIONAL THERAPISTS
}

\begin{abstract}
BACKGROUND AND AIMS

Low Back Pain (LBP) is considered as the second leading source of musculoskeletal disorders, affecting $85 \%$ individuals worldwide at some point in their life time. Numerous studies estimated Occupational Therapists have predominant risk of developing LBP due to nature of their daily activities therefore the aim of this study is to evaluate the effectiveness of lumbar stabilizing exercises among Occupational Therapists with non-specific LBP.
\end{abstract}

\section{METHODOLOGY}

A single blind, RCT was conducted at Dr. Ziauddin Hospital included 30 Occupational Therapists divided in to Group A and B that received lumbar stabilization and general extension exercises respectively. Both the groups received 4 weeks of intervention on 6 days/week. Data was collected Pre and Post the treatment on Numeric Rating Scale and Oswestry LBP Disability Questionnaire.

\section{RESULTS}

Both the groups showed significant results $(p<0.05)$, however Group A showed marked reduction in pain and disability in comparison to Group B that showed moderate decrease in both outcome measures.

\section{CONCLUSION}

Lumbar stabilization exercises are more effective to reduce LBP as compared to general extension exercises. Multi-center trials are recommended in the management of LBP in healthcare professionals to prevent musculoskeletal disorders.

\section{KEYWORDS}

Low Back Pain, Mechanical, Postural, Occupational Therapist, Spinal Column, Disorders.

\author{
Jaza Rizvi \\ Lecturer \\ Ziauddin College of Rehabilitation \\ Sciences \\ Ziauddin University \\ tyhil1992@gmail.com

\section{Neelum Zehra} \\ HOD Occupational Therapy \\ Ziauddin College of Rehabilitation \\ Sciences \\ Ziauddin University \\ neelumzehra@gmail.com
}

\section{Hira Masood}

Senior Occupational Therapist KDSP

hiramasood.90@gmail.com

[Rizvi J, Zehra N, Masood H.Effectiveness of Lumbar Stabilization Exercises in Non-specific Low Back Pain among Occupational Therapists. Pak. J. rehab. 2019;8(1):43-48] 


\section{INTRODUCTION}

According to World Health Organization (WHO), mechanical low back pain (LBP) is considered as the second leading source of musculoskeletal disorders', affecting $85 \%$ of all individuals worldwide at some point in their life time. The occurrence of severe LBP, in particular mechanical type is only $14 \%$, persisting for $>2$ weeks. Within all cases of the disorder, $70 \%$ are caused due to lumbar sprain/strain while only $10 \%$ occurred due to degenerative changes caused by aging while the specific LBP prevalence is $4 \%$ due to herniated disks ${ }^{1-2}$. In addition, $4 \%$ of the individuals suffered LBP due to osteoporosis or compression fractures of spine while only $3 \%$ suffers the pain due to spinal stenosis ${ }^{1-2}$. It has been estimated that LBP represents the utmost source of disability concomitant with the work related injury in individuals aged $<45$ years' ${ }^{1}$. Although, mechanical LBP among health care professionals can be caused by most prevailing factors such as obesity, age and poor posture etc ${ }^{3}$. Other contributing factors including physical activities like lifting, twisting or bending may worsen the symptoms which may be relieved by rest ${ }^{1-3}$. These consequences are severe and may lead to a negative economic impact, which includes an increased absenteeism from workplace and loss of productiveness ${ }^{4}$. Nevertheless, most episodes of mechanical LBP have a favorable prognosis but the recurrence of pain within a year is common ${ }^{5}$.

Numerous studies estimated that workers involved in healthcare comprises of physical and occupational therapists have predominant risk in developing LBP, either mechanical or specific due to prolonged sitting, standing, stooping and repetitive low and high risks lifting activities due to nature of their daily activities $^{1-5}$. It was also concluded that occupational therapists have substantial degrees of musculoskeletal pain and work-related disorders due to the physical demand of their role in health care ${ }^{6}$. However, limited researches investigated LBP among occupational therapists or its impact in the nature of job ${ }^{5-6}$. The studies also predicted the certain degree of risk incurred from the usage of computer among occupational therapists and other health care professionals who usually spent approximately 16-20 hours per week on the computer which has also become a reason of an increased risk of the disorder ${ }^{7}$. Yet physical demands of the work are considerable, particularly with activities associated with the rehabilitation of people with disabilities ${ }^{8}$.

According to the Bureau of Labor Statistics, the clinicians or healthcare workers attend patients with intricate medical conditions that may require assistance in daily living activities or gait training ${ }^{8-9}$. The inpatient and outpatient therapists often attend patients that entail appropriate dosage of manual therapy, relocations or splint fabrications that may leads to risk of developing LBP. All these aspects potentially lead to the work-related musculoskeletal disorders with an increased rate of 2.61 times $^{6-9}$.
Recent studies have emphasized that advance therapeutic exercises have played a vital role in decreasing LBP but also in strengthening muscles play pivotal role in maintaining posture that supports the spine in standing, bending and lifting activities ${ }^{10}$. These core muscles are responsible to extend and flex the spine in order to balance the lifted weight therefore; the muscle strength of extensor muscle is a crucial factor for the spinal stability ${ }^{10-11}$.

Several studies reported different therapeutic management approaches for the treatment of LBP although, these management strategies are modified with advances time ${ }^{12}$. At present practice, stabilizing exercises are incorporated in improving muscle strength, endurance and flexibility of core muscles. It has been documented that these exercises are aided to strengthen spinal segments, enhance the stability thereby prevents LBP $1^{1-12}$. Moreover, it is prescribed that the screening of disorder and its episodes requires a detailed evaluation of patient's condition to integrate appropriate management strategy. Consecutively, there is lack of research evidence on this regard as only incidence of lower back pain among occupational therapists and other health professionals except for physiotherapists has been investigated. Moreover, the evidence related to lumbar stabilization exercises in LBP among occupational therapists has not been inspected and remains unclear. Thus, the aim of this study is to evaluate the effectiveness of lumbar stabilizing exercises among occupational therapists with non-specific LBP. Furthermore, this research will be served as the basis for the future use of described therapeutic protocol among the health care professionals in order to prevent work-related injuries, musculoskeletal pain and disorders.

\section{METHODOLOGY}

\section{Study Design}

A Single-Blind, Randomized Control Trial.

\section{Study Setting and Participants}

The study was conducted at rehabilitation sciences department of primary and tertiary care hospitals considering occupational therapists with non-specific LBP.

\section{Duration of Study}

Study was completed within the duration of 6 months.

\section{Sample Size}

A total sample of 30 participants was recruited in the study.

\section{Sampling Technique}

A sample of 30 participants were randomly divided into Group A and B through toss a coin method of simple random sampling. Patients who received heads were enrolled in Group-A $(n=15)$ whereas all participants who received tails were allocated to 
Group-B (n=15).

\section{Inclusion Criteria}

Both male and female participants aged $25-45$ years who had non-specific LBP of $>6$ weeks with no radicular symptoms were included.

\section{Exclusion Criteria}

Participants who had pregnancy, radiating pain, inflammatory disease, nerve compression, fracture or dislocations or any sign of red flags were excluded.

\section{Data Collection Tool}

The data was collected on two outcome measures for the assessment of pain and disability due to LBP:

- The Numeric Rating Scale (NRS), a subjective measure of pain. The scale is comprised of the scoring of 0 to 10, confined with "no pain" to "worst pain"13.

- The Oswestry Disability Index (ODI) is a questionnaire that comprised of ten aspects related to pain intensity, activities related to lifting, ability to walk, taking care, sit and stand, social life, quality of sleep and travelling. Each aspect has a scoring of $0-5$ in which the first statement indicates the least amount of disability and last statement represents severe disability. The total scoring of all the questions is summed up on the index of $0 \%$ to $100 \%{ }^{14}$.

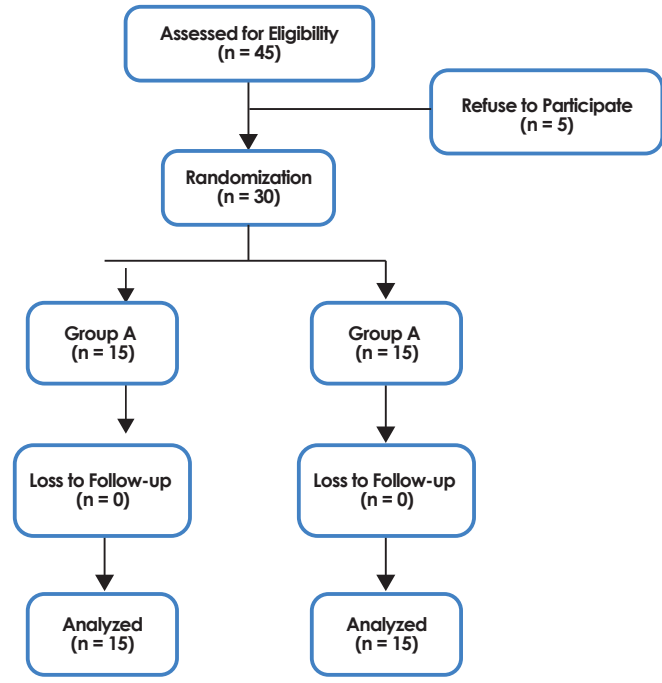

Figure 1. Randomization of Group A and B

\section{Data Collection Procedure}

A total of 30 participants were randomized in to Group A and B, 15 participants in each group respectively as shown in Figurel.

Each participant was given informed consent prior to intervention to ensure clear understanding regarding protocol. Participants of Group-A received lumbar stabilization exercises consists of antero-posterior pelvic tilts, core contraction, straight leg lifts, single leg straight and squares and forward lunges. Preliminary to exercises, each participant was asked to perform 5 minutes warm-up then perform 3 sets of these exercises, each set was consisted of 10 repetitions for the duration of 20 minutes. Afterwards, participants were asked to perform 5 minutes of cool-down. The total duration of protocol lasted for 30 minutes.

On the other hand, Group-B participants received general extension exercises for pain relieving consisted of prone lying relaxation, elbow press, press ups and backward bending. Similarly, before the exercises each participant was asked to perform 5 minutes warm-up followed by 3 sets of these exercises with 10 repetitions in each set for the duration of 20 minutes followed by 5 minutes cool-down. The total duration of protocol lasted for 30 minutes.

Both the groups received intervention 6 days/week for 4 weeks while the data was collected pre and post the treatment on NRS and ODI. Moreover, therapist taught the respected exercises to both the groups at lst day of intervention and prescribed follow-up for the assigned days using pamphlet and reminders at their feasible time (tele-medicine). Furthermore, therapist visited participants of both groups once in a week for 4 weeks to assess the follow-up of prescribed exercises.

\section{Data Analysis}

Data was entered and analyzed on IBM SPSS Statistics (Statistical Package for the Social Sciences) version 20. The frequency and standard deviations of the demographic data was determine through descriptive statistics while paired t-test and independent t-test were applied as an inferential statistics within and in between the groups respectively.

\section{RESULTS}

Total 30 participants, 15 in each group respectively completed the protocol with mean age of $26.73 \pm 1$. 62 in Group A with 2 males and 13 female subjects correspondingly whereas in Group B, 5 participants were male and 10 were females with mean age of $28.33 \pm 3.06$. Participants were assessed on NRS and ODI at 4 weeks pre and post intervention; moreover no loss of follow-up has been reported. The demographic characteristics of participants are shown in Table 1.

\begin{tabular}{|c|c|c|}
\hline & $\begin{array}{c}\text { GROUP-A }(n=15) \\
\text { Mean } \pm \text { S.D. }\end{array}$ & $\begin{array}{c}\text { GROUP-B }(n=15) \\
\text { MeantS.D. }\end{array}$ \\
\hline $\begin{array}{c}\text { Age } \\
\text { (years) }\end{array}$ & $26.73 \pm 1.62$ & $28.33 \pm 3.06$ \\
\hline Gender & $\begin{array}{c}2 \text { Males } \\
13 \text { Females }\end{array}$ & $\begin{array}{c}5 \text { Males } \\
10 \text { Females }\end{array}$ \\
\hline Height & $156.06 \pm 3.32$ & $158.26 \pm 3.89$ \\
\hline Weight & $61.13 \pm 5.40$ & $63.66 \pm 5.32$ \\
\hline
\end{tabular}


On Paired T-test analysis, Group-A showed significant decrease in pain on NRS and disability due to LBP on ODI in 4-weeks intervention. Similarly, Group-B reported significant results $(\mathrm{p}<0.05)$ but the pain and disability were moderately reduce in comparison to Group A as shown in Table 2.

\begin{tabular}{|c|c|c|c|c|}
\hline \multicolumn{5}{|c|}{ Table 2. Within the Group PrePost Mean Differences } \\
\hline Intervention & $\begin{array}{l}\text { Pre NRS } \\
\text { Mean } \pm S . \\
\text { D }\end{array}$ & $\begin{array}{c}\text { Post NRS } \\
\text { Mean } \pm S . \\
\text { D }\end{array}$ & $\begin{array}{c}\text { Mean } \\
\text { Difference }\end{array}$ & $\begin{array}{c}{ }^{* P} \text { - } \\
\text { value }\end{array}$ \\
\hline Group-A & $6.60 \pm 0.98$ & $4.6 \pm 0.61$ & $2.0 \pm 0.37$ & \multirow{4}{*}{$<0.05$} \\
\hline Group-B & $6.40 \pm 1.1$ & $5.2 \pm 1.0$ & $1.2 \pm 0.1$ & \\
\hline Group-A & $30.1 \pm 5.9$ & $24.9 \pm 5.6$ & $5.2 \pm 0.3$ & \\
\hline Group-B & $33.9 \pm 6.27$ & $30.2 \pm 5.58$ & $3.7 \pm 0.69$ & \\
\hline
\end{tabular}

Moreover, difference between Group A and B was determined through Independent T-test after 4 weeks of protocol. The differences between the two groups were analyzed using post mean values of components. In general, both the groups showed significant results $(p<0.05)$, however Group A showed marked reduction in pain and disability in comparison to Group B that showed moderate decrease in both the outcome measures as shown in Figurel.

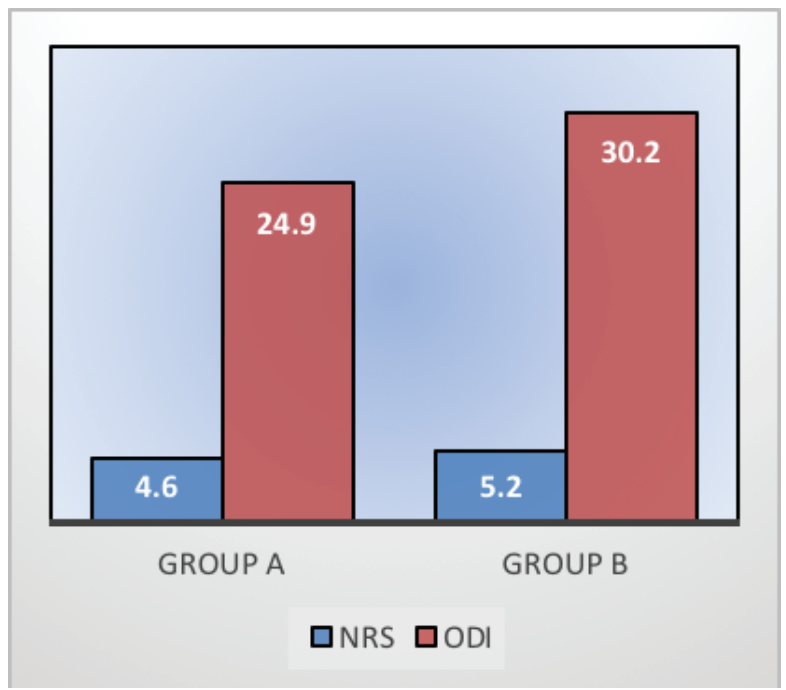

Figure 1. Comparison of post mean values of Group $A$ and $B$.

\section{DISCUSSION}

The study findings revealed that lumbar stabilization exercises and extension exercises were both effective to reduce non-specific LBP and disability in occupational therapists. However, lumbar stabilization exercises are clinically more significant to reduce the health-related outcomes in comparison to general extension exercises. These results were consistent with the study of Inani SB ${ }^{15}$ which concluded core stability exercises are effective in reducing pain, disability and activation of core muscles. Moreover, these exercises are beneficial to activate the core muscles and reduce the lumbar spine instability ${ }^{16}$. Therefore, it has been established that core stabilization exercises not only improves the ability of core muscles but also improved function in patients with chronic non-specific $\mathrm{LBP}^{17}$. Similar to the context, several studies reported significant reduction in LBP by aiding stabilization of lumbar spine during the performance of functional tasks ${ }^{16-18}$. These outcomes reported significantly decrease in LBP among the subjects. On the contrary, another study established favorable evidence of electrotherapeutic modalities in management of chronic non-specific LBP19. The study revealed clinically marked reduction in pain on Numeric Rating Scale (NRS) and disability on Roland-Morris Disability questionnaire. Furthermore, our study observed a significant reduction between the baselines and 4th weeks on pain intensity and disability of participants that revealed the LBP management in effective time frame by a sole intervention. In accordance, other authors which reported that specific lumbar stabilizing therapy that reduced the pain intensity of lower back and pelvic girdle either used the protocol as an adjuvant treatment or with the combination ${ }^{20}$. A previous review concluded that majority of studies favors lumbar stabilization exercises in combination to different exercises while recent studies implying the intervention as a sole intervention to reduce the burden of disability caused by LBP22-23. Several studies reported the disability using Oswestry Disability Index (ODI) subsequent to lumbar stabilization exercises that revealed approximately $20-30 \%$ reduction in disability ${ }^{14}$. Therefore, ODI is an effective tool to measure disability. However, another study indicated that general extension is easier to perform and have long-term effect on LBP while stabilizing exercises have short benefit to pain and disability ${ }^{24}$. In contrast, a study concluded that stabilization exercises have long-term benefit in chronic back pain and disability in comparison to extension exercises ${ }^{24}$. A study conducted by Lee et $\mathrm{al}^{25}$ reported the significance of local and global muscle stabilizers that directed the strength in different exercise positions included multifidus, transverse and rectus abdominus, obliqus internus and externus, thoracic longissimus. These muscles support the body especially during acquired supine and prone positions respectively $22-24$. Moreover, it was demonstrated that stabilizing exercises have efficacy to increment in muscle strength and balance as well as in relieving of pain ${ }^{24-25}$. On the contrary, number of studies have validated that isometric exercises in LBP has hypogelsic effect by activating the secretion of opioids that leads to reduction in the pain perception ${ }^{25}$. However, it was also concluded that either the types of exercises carried in supine, prone or combination of positions are beneficial in reducing the disability and pain in patients with chronic non-specific LBP. 
Although, still there is a debate in terms of most effective back pain management and how it effects changes to the musculoskeletal system ${ }^{19-22}$. Therefore, multicenter randomized controlled trials are recommended to evaluate the efficacy of exercise interventions. Further, it is important for the clinicians to have a complete understanding of underlying cause of LBP in order to have adequate information regarding pros and cons of pain and disability. However, the evaluation of core can be a difficulty for therapists; therefore a proper training before the facilitation of exercises is recommended to objectively quantify the individual's core strength ${ }^{21-24}$. The limitations in this study are small size that may not be implied for generalizability of research findings, therefore large sample size while considering different health professionals is recommended. Further, the patients enrolled in the study were relatively younger and didn't have significant disability and that may not conclude the generalize impact of lumbar stabilization exercises. Therefore, the study failed to predict the prolonged effects of an intervention. Despite, the strength of core muscles and exercises were not monitored directly, therefore several patients faced certain difficulties in performing the exercises in a routine. Moreover, we have introduced the concept of tele-medicine that may serves as an innovative approach in the protocol. Yet, the disparity has been observed among participants in terms of performance and also the results may not reflect the overall strength of core muscles. Thus, it is suggested to perform the exercise under investigators supervision in order to get desired outcomes and device a management strategy for an effective LBP treatment.In conclusion, lumbar stabilization exercise is a crucial and effective strategy in treating LBP either in chronic or acute stage. Thus, it is recommended to apply the intervention with an appropriate dosage, intensity and repetitions for improving in patient's health related outcomes. Also, clinicians must seek subjective and objective measurement of muscles to prescribe better treatment approach for patients for clinically significant results.

\section{CONCLUSION}

It was concluded that lumbar stabilization exercises are more effective to reduce non-specific as compared to general extension exercises. However, multi-center large scale trials are recommended in the management of LBP among different healthcare professionals to prevent musculoskeletal injuries and disorders as well as enhance the occupational performance.

\section{REFERENCES}

[1] Maher C, Underwood M, Buchbinder R. Non-specific low back pain. The Lancet. 2017;389(10070):736-47.

[2] Oliveira CB, Maher CG, Pinto RZ, Traeger AC, Lin CW, Chenot JF, van Tulder M, Koes BW. Clinical prac- tice guidelines for the management of non-specific low back pain in primary care: an updated overview. Eur Spine J. 2018;27(11):2791-803.

[3] Scott Will J, Bury DC, Miller JA. Mechanical Low Back Pain. Am Fam Phys. 2018;98(7).

[4] Aremu AB, Olaniyan LT. Prevalence and factors associated with low back pain among healthcare workers in kibuli Muslim hospital kampala, Uganda.

[5] Rajsekhar H, Latha PS, Kumar PB. A Comparative Study to Find the Effect of Stabilization and Extension Exercises on Patients with Sub-Acute Non-Specific Low Back Pain. Int J Physiother Res. 2017;5(4):2282-88. [6] Suni JH, Rinne M, Kankaanpää $M$, Taulaniemi A, Lusa S, Lindholm H, Parkkari J. Neuromuscular exercise and back counselling for female nursing personnel with recurrent non-specific low back pain: study protocol of a randomised controlled trial (NURSE-RCT). BMJ. 2016;2(1):e000098.

[7] Ye S, Jing Q, Wei C, Lu J. Risk factors of non-specific neck pain and low back pain in computer-using office workers in China: a cross-sectional study. BMJ open. 2017;7(4):e014914.

[8] Rodrigues-De-Souza DP, Fernández-De-Las-Peñas C, Martín-Vallejo FJ, Blanco-Blanco JF, Moro-Gutiérrez $L$, Alburquerque-Sendín F. Differences in pain perception, health-related quality of life, disability, mood, and sleep between Brazilian and Spanish people with chronic non-specific low back pain. BJPT. 2016(AHEAD):0-

[9] Cullen KL, Irvin E, Collie A, Clay F, Gensby U, Jennings PA, Hogg-Johnson S, Kristman V, Laberge $M$, McKenzie D, Newnam S. Effectiveness of workplace interventions in return-to-work for musculoskeletal, pain-related and mental health conditions: an update of the evidence and messages for practitioners. J Occup Rehabil. 2018;28(1):1-5.

[10] Salavati M, Akhbari B, Takamjani IE, Bagheri H, Ezzati K, Kahlaee AH. Effect of spinal stabilization exercise on dynamic postural control and visual dependency in subjects with chronic non-specific low back pain. J Bodyw Mov Ther. 2016;20(2):441-8 [11] Searle A, Spink M, Ho A, Chuter V. Exercise interventions for the treatment of chronic low back pain: a systematic review and meta-analysis of randomised controlled trials. Clin Rehabil. 2015;29(12):1155-67.

[12] Ali S, Sajjad AG, Keramat KU, Darian H. Chronic Low Back Pain; Effects of the Lumbar Stabilization Exercises on Pain, Range of Motion and Functional Disability in the Management. TPMJ. 2017;24(4).

[13] Lee JJ, Lee MK, Kim JE, Kim HZ, Park SH, Tae JH, Choi SS. Pain relief scale is more highly correlated with numerical rating scale than with visual analogue scale in chronic pain patients. Pain Physician. 2015;18(2):E195-200.

[14] Chiarotto A, Maxwell LJ, Terwee CB, Wells GA, Tugwell P, Ostelo RW. Roland-Morris Disability Questionnaire and Oswestry Disability Index: which has better measurement properties for measuring physical functioning in nonspecific low back pain? Systematic review and meta-analysis. Phys Ther. 2016:96(10):1620-37.

[15] Inani SB, Selkar SP. Effect of core stabilization 
exercises versus conventional exercises on pain and functional status in patients with non-specific low back pain: a randomized clinical trial. Journal of back and musculoskeletal rehabilitation. 2013;26(1):37-43.

[16] Akhtar MW, Karimi H, Gilani SA. Effectiveness of core stabilization exercises and routine exercise therapy in management of pain in chronic non-specific low back pain: A randomized controlled clinical trial. Pak JMed Sci. 2017;33(4):1002.

[17] Puntumetakul R, Chalermsan R, Hlaing SS, Tapanya W, Saiklang $P$, Boucaut R. The effect of core stabilization exercise on lumbar joint position sense in patients with subacute non-specific low back pain: a randomized controlled trial. Phys Ther. 2018;30(1 1):1390-5.

[18] Gordon R, Bloxham S. A systematic review of the effects of exercise and physical activity on non-specific chronic low back pain. InHealthcare 2016 Jun (42, p. 22). Multidisciplinary Digital Publishing Institute. [19] Rahman D, Jahan I, Uddin S, Uddin Z, Islam S. Comparison the Effectiveness of Manual Therapy, Electrotherapy and Core Stability Exercise vs Electrotherapy, Traction and Traditional Exercise for Acute Low Back Pain. MOJ Orthop Rheumatol. 2015;2(2):00042.
[20] Shipton EA. Physical therapy approaches in the treatment of low back pain. Pain Ther. 2018;7(2):127-37.

[21] Moon HJ, Choi KH, Kim DH, Kim HJ, Cho YK, Lee $\mathrm{KH}$, Kim JH, Choi YJ. Effect of lumbar stabilization and dynamic lumbar strengthening exercises in patients with chronic low back pain. Annals of rehabilitation medicine. 2013;37(1):110.

[22] Shahvarpour A, Gagnon D, Preuss R, Henry SM, Larivière C. Trunk postural balance and low back pain: reliability and relationship with clinical changes following a lumbar stabilization exercise program. Gait Posture. 2018;61:375-81.

[23] Yoon JO, Kang MH, Kim JS, Oh JS. Effect of modified bridge exercise on trunk muscle activity in healthy adults: a cross sectional study. BJPT. 2018;22(2):161-7.

[24] De Blaiser C, Roosen P, Willems T, Danneels L, Bossche LV, De Ridder R. Is core stability a risk factor for lower extremity injuries in an athletic population? A systematic review. Physical therapy in sport. 2018;30:48-56..

[25] Wáng YX, Wu AM, Santiago FR, Nogueira-Barbosa $\mathrm{MH}$. Informed appropriate imaging for low back pain management: A narrative review.JOT.2018;15:21-34. 